\title{
Spin dependence of the scattering of protons from halo nuclei
}

\author{
R. Crespo, ${ }^{*}$ J.A. Tostevin, and R.C. Johnson \\ Department of Physics, University of Surrey, Guildford, Surrey, GU2 5XH, United Kingdom
}

(Received 22 December 1994)

\begin{abstract}
The optical potential and observables for proton- ${ }^{8} \mathrm{He}$ elastic scattering are studied within the single scattering approximation to the Kerman, McManus, and Thaler (KMT) multiple scattering expansion. Calculations are performed for proton $-{ }^{8} \mathrm{He}$ elastic scattering at $72 \mathrm{MeV} / \mathrm{nucleon}$, for which there are available experimental data, and also at $200 \mathrm{MeV} /$ nucleon incident energy. It is shown that the calculated elastic scattering observables are modified significantly by the inclusion of the proton- ${ }^{8} \mathrm{He}$ spin dependence arising from the nucleon-nucleon amplitude. The roles of the core and valence nucleon contributions to this spin dependence are clarified.
\end{abstract}

PACS number(s): 24.10.Ht, 24.70.+s, 25.40.Cm

\section{INTRODUCTION}

With the advent of beams of radioactive nuclei of high energy [1] from fragmentation reactions there is increasing interest in studies of the structure of light nuclei far from stability, and of the extent to which these structures can be elucidated through the analysis of scattering and reaction data. Root mean square ( $\mathrm{rms}$ ) radii of the matter densities of the unstable nuclei have been deduced from interaction $[2,3]$ and reaction [4] cross section measurements.

One- and two-neutron halos have now been observed in several light neutron-rich nuclei, such as ${ }^{11} \mathrm{Be}$ and ${ }^{11} \mathrm{Li}$. These are characterized by a striking departure of the interaction radii from the $A^{1 / 3}$ rule [5]. Halo nuclei are also characterized by having extremely small one- and/or twoneutron separation energies. The $n$ separation energy is $0.5 \mathrm{MeV}$ in the case of ${ }^{11} \mathrm{Be}$ and the $2 n$ separation energy is $0.3 \mathrm{MeV}$ for ${ }^{11} \mathrm{Li}$. Matter radii have also been deduced for the sequence of helium isotopes ${ }^{4,6,8} \mathrm{He}[3,6]$. The observed two-neutron separation energies are $0.97 \mathrm{MeV}$ for ${ }^{6} \mathrm{He}$ and $2.13 \mathrm{MeV}$ for ${ }^{8} \mathrm{He}$.

Of the nuclei mentioned, ${ }^{8} \mathrm{He}$ is a particularly good candidate to study halo effects upon the reaction mechanism. The structure of ${ }^{8} \mathrm{He}$ is thought to be understood and, to a good approximation, comprises a closed $j=3 / 2$ valence neutron subshell and an alpha particle core. Thus the ${ }^{8} \mathrm{He}$ ground state has spin zero, simplifying the possible spin-dependent terms in the ${ }^{8} \mathrm{He}$-target optical potential.

Much theoretical effort has now been invested in assessing the importance of the halo effects on the total reaction cross section and on scattering differential cross section angular distributions. Within the context of ionion collisions, the study of the ${ }^{11} \mathrm{Li}+{ }^{12} \mathrm{C}$ system [7] has shown it to behave in a very similar way to ${ }^{12} \mathrm{C}+{ }^{12} \mathrm{C}$ but

\footnotetext{
${ }^{*}$ On leave from Departamento de Física, Instituto Superior Técnico, Lisboa, Portugal.
}

with the need phenomenologically [8] for central potentials of extended range. There remain, however, significant discrepancies between the data and their theoretical description in terms of an underlying ${ }^{11} \mathrm{Li}$ structure and constituent cluster-target interactions [9].

In the case of ion-nucleon scattering, measurements of the elastic scattering cross section angular distributions of the lithium isotopes ${ }^{6,9,11} \mathrm{Li}$ from protons at 60 $\mathrm{MeV} /$ nucleon [10] and of the helium isotope ${ }^{8} \mathrm{He}$ from protons at $72 \mathrm{MeV} /$ nucleon [11] have been undertaken. An important possibility for ion-nucleon systems is that analyzing power measurements (in inverse kinematics) could be made in the future by use of a polarized proton target. The nucleon-ion spin dependence, the spin-orbit interaction for a spinless ion, is expected to have significant effects at the energies of current experimental interest. In this paper we provide a first assessment of the effects on the proton- ${ }^{8} \mathrm{He}$ elastic scattering observables due to the intrinsic spin dependence of the free nucleonnucleon $(N N)$ amplitude. In particular, we attempt to clarify the spin sensitivity, due to the extended neutron halo of ${ }^{8} \mathrm{He}$, in calculated cross sections and analyzing powers. In doing so we clarify the extent to which spin measurements might allow one to probe further the structure of the valence nucleons.

The phenomenological analysis of the proton-Li scattering data reported in Ref. [10] did include a nucleonion spin-orbit interaction. However, its significance for describing the data was not clarified. On the other hand, the theoretical calculations so far reported for elastic ${ }^{9,11} \mathrm{Li}$-proton [12] and ${ }^{8} \mathrm{He}$-proton [13] scattering, based on a Glauber optical potential, assume a parametrized zero-range effective $N N$ transition amplitude and neglect its explicit spin dependence. Nevertheless, these calculations reveal that the elastic cross section angular distributions show sensitivity to the assumed single-particle density of the incident ion, although some features of the experimental data, such as the reduced magnitude of the empirical cross section angular distribution for $p^{-11} \mathrm{Li}$ scattering in comparison with that for the other isotopes, are not reproduced [12]. 
The present analysis is carried out within the microscopic multiple scattering formalism of Kerman, McManus, and Thaler (KMT) [14]; the essental inputs are thus the free nucleon-nucleon transition amplitude and the projectile $\left({ }^{8} \mathrm{He}\right)$ wave function. Calculations will be presented for $p^{8} \mathrm{He}$ scattering at $72 \mathrm{MeV} /$ nucleon, where cross section data are already available, and at 200 $\mathrm{MeV} /$ nucleon incident energy. Applications of the multiple scattering treatment of the optical potential have increased considerably in sophistication during the last few years [15-18] and careful treatments of the firstand second-order terms of the multiple scattering series have been carried out. The multiple scattering approach, which expands the nucleon-target optical potential in terms of a realistic free $N N$ transition amplitude, evaluated at an appropriate $N N$ relative energy parameter, is best suited for nucleon incident energies of order 100 $\mathrm{MeV} \leq E_{0} \leq 400 \mathrm{MeV}$. The method has, however, recently been applied with some success for the description of elastic nucleon scattering data from a wide range of targets at $65 \mathrm{MeV}$ [19].

The nonlocality of the $N N$ amplitude means that the optical potential and the solution of the scattering problem are most conveniently carried out in momentum space. We include both the central and spin-orbit optical potential components. In the present work the $N N$ amplitude is calculated from the Paris potential [20-22]. Particular attention will be paid to the importance of the spin dependence on the calculated elastic scattering observables and the implications for analyses aimed at a determination of the halo structure of such light neutron rich nuclei.

\section{OPTICAL POTENTIAL}

The first-order term of the KMT optical potential for nucleon scattering from a target of mass $A$ is given by the expression $[14,16]$

$$
U=\frac{A-1}{A} \sum_{n=1}^{A}\left\langle\phi_{0}\left|t_{0 n}^{f}(\omega)\right| \phi_{0}\right\rangle .
$$

Here $\phi_{0}$ is the target nucleus wave function and $t_{0 n}^{f}(\omega)$ is the $N N$ transition operator describing the free scattering of the incident $(0)$ and struck $(n)$ target nucleon with an energy parameter $\omega$. This transition amplitude satisfies the integral equation

$$
t_{0 n}^{f}(\omega)=v_{0 n}+v_{0 n} g(\omega) t_{0 n}^{f}(\omega),
$$

where $v_{0 n}$ is the assumed free space $N N$ interaction. The intermediate state propagator $g(\omega)$ is therefore

$$
g(\omega)=\frac{1}{\omega^{+}-K_{0 n}},
$$

where $K_{0 n}$ is the kinetic energy operator for the relative motion of the active $N N$ pair. Medium effects arising from distortions due to the struck nucleon binding potential have been neglected in the propagator [17]. The energy parameter is $\omega=E_{0}-\hbar^{2} \hat{\mathcal{P}}^{2} / 4 m$, where $\hat{\mathcal{P}}$ is the momentum operator for the motion of the center of mass (c.m.) of the interacting $N N$ pair [17], and $m$ is the nucleon mass. $E_{0}$ is the incident nucleon energy in the nucleon-target $(N A)$ c.m. frame, thus $E_{0}=\hbar^{2} k_{0}^{2} / 2 \mu_{N A}$ with $\mu_{N A}$ the nucleon-target reduced mass.

The second-order term of the multiple scattering expansion, which takes into account Pauli blocking medium effects, has been investigated elsewhere in considerable detail [17]. These Pauli blocking corrections modify the optical potential only in the nuclear interior [23] and are not considered explicitly here.

The matrix elements of the optical potential are developed in momentum space as

$$
\left\langle\vec{k}^{\prime}|U| \vec{k}\right\rangle=\frac{A-1}{A} \sum_{n=1}^{A}\left\langle\vec{k}^{\prime} \phi_{0}\left|t_{0 n}^{f}(\omega)\right| \vec{k} \phi_{0}\right\rangle .
$$

Introducing the variables $\vec{P}=\left(\vec{p}+\vec{p}^{\prime}\right) / 2$ and $\vec{Q}=$ $\left(\vec{k}+\vec{k}^{\prime}\right) / 2$, the mean values of the scattered and struck nucleon momenta, then

$$
\begin{aligned}
\left\langle\vec{k}^{\prime}|U| \vec{k}\right\rangle= & \frac{A-1}{A} \sum_{n=1}^{A} \int d^{3} P\left\langle\phi_{0} \mid \vec{P}-\vec{q} / 2\right\rangle\left\langle\vec{\kappa}^{\prime}\left|t_{0 n}^{f}(\omega)\right| \vec{\kappa}\right\rangle \\
& \times\left\langle\vec{P}+\vec{q} / 2 \mid \phi_{0}\right\rangle,
\end{aligned}
$$

where $\vec{q}=\vec{k}^{\prime}-\vec{k}$ is the momentum transfer and the initial and final $N N$ relative momenta are

$$
\vec{\kappa}=\frac{1}{2}(\vec{k}-\vec{P}-\vec{q} / 2), \quad \vec{\kappa}^{\prime}=\frac{1}{2}\left(\vec{k}^{\prime}-\vec{P}+\vec{q} / 2\right) .
$$

The energy parameter $\omega$ can now be written

$$
\omega=E_{0}-\frac{\hbar^{2}}{4 m}|\vec{Q}+\vec{P}|^{2}
$$

In this momentum space form, Eq. (5), a $N N$ transition amplitude from realistic $N N$ interaction models can be included. With the convention that plane waves are normalized such that

$$
\langle\vec{r} \mid \vec{k}\rangle=(2 \pi)^{-3 / 2} \exp (i \vec{k} \cdot \vec{r}),
$$

then the antisymmetrized transition amplitude is related to the $N N$ scattering amplitude $M$ by the relation

$$
\left\langle\vec{\kappa}^{\prime}\left|t_{0 n}^{f}(\omega)\right| \vec{\kappa}\right\rangle=-\frac{\hbar^{2}}{4 \pi^{2} \mu}\left\langle\vec{\kappa}^{\prime}|M| \vec{\kappa}\right\rangle,
$$

with $\mu$ the $N N$ reduced mass, and where [24]

$$
\begin{aligned}
M= & \mathcal{A}+\mathcal{B}\left(\vec{\sigma}_{0} \cdot \hat{n}\right)\left(\vec{\sigma}_{n} \cdot \hat{n}\right)+\mathcal{C}\left(\vec{\sigma}_{0}+\vec{\sigma}_{n}\right) \cdot \hat{n} \\
& +\mathcal{D}\left(\vec{\sigma}_{0} \cdot \hat{m}\right)\left(\vec{\sigma}_{n} \cdot \hat{m}\right)+\mathcal{E}\left(\vec{\sigma}_{0} \cdot \hat{\ell}\right)\left(\vec{\sigma}_{n} \cdot \hat{\ell}\right) \\
& +\mathcal{F}\left[\left(\vec{\sigma}_{0} \cdot \hat{\ell}\right)\left(\vec{\sigma}_{n} \cdot \hat{m}\right)+\left(\vec{\sigma}_{n} \cdot \hat{m}\right)\left(\vec{\sigma}_{0} \cdot \hat{\ell}\right)\right] .
\end{aligned}
$$

Here $\hat{n}=\vec{\kappa} \times \vec{\kappa}^{\prime} /\left|\vec{\kappa} \times \vec{\kappa}^{\prime}\right|, \hat{\ell}=\left(\vec{\kappa}^{\prime}+\vec{\kappa}\right) /\left|\vec{\kappa}^{\prime}+\vec{\kappa}\right|$, and $\hat{m}=$ $\hat{\ell} \times \hat{n}$ are the unit vectors defined by the $N N$ scattering plane.

Of the KMT amplitudes $\mathcal{A}, \mathcal{B}, \mathcal{C}, \mathcal{D}, \mathcal{E}$, and $\mathcal{F}$, only $\mathcal{A}$ 
and $\mathcal{C}$ are required in the context of the present work. In calculations these amplitudes are expressed as complex functions of relative energy $\omega$, momentum transfer $\vec{q}=$ $\left(\vec{\kappa}^{\prime}-\vec{\kappa}\right)$, and total $N N$ momentum $\overrightarrow{\mathcal{Q}}=\left(\vec{\kappa}^{\prime}+\vec{\kappa}\right) / 2$ in their c.m. frame. Each amplitude depends on isotopic spin in the form

$$
\mathcal{A}\left(\omega, \vec{\kappa}^{\prime}, \vec{\kappa}\right)=\mathcal{A}(\omega, \vec{q}, \overrightarrow{\mathcal{Q}})=\mathcal{A}_{0}+\mathcal{A}_{\tau}\left(\vec{\tau}_{0} \cdot \vec{\tau}_{n}\right)
$$

\section{TARGET NUCLEUS MODEL}

To describe the ${ }^{8} \mathrm{He}$ ground state we take the five-body cluster orbital shell model approximation (COSMA) wave function proposed by Zhukov et al. [25]. The wave function is written as the product of an $\alpha$ particle core wave function with a function which describes the motion of the valence neutrons, each assumed to occupy a $p_{3 / 2}$ state relative to the core. The core and valence nucleons are assumed to occupy harmonic-oscillator single-particle states with the oscillator parameter for the core nucleons chosen to reproduce the alpha particle matter rms radius $1.45 \mathrm{fm}$. The total wave function thus contains only one adjustable parameter, the oscillator parameter for the valence nucleons, which is chosen to reproduce the rms matter radius of ${ }^{8} \mathrm{He}$ of $2.52 \mathrm{fm} \mathrm{[3]}$.

A simple parametrized Gaussian form for the core and valence nucleon densities is also presented in [25]. When compared with the full COSMA model approximation this density produces the same rms matter radius and accurate values for $r_{v}=3.14 \mathrm{fm}$ and $r_{p}=1.69 \mathrm{fm}$, the mean distances of the valence neutrons, and of a point proton from the ${ }^{8} \mathrm{He}$ c.m., respectively. The Fourier transforms of these densities are, for the $\alpha$ core,

$$
\rho_{c}(q)=4 \exp \left(-b_{c}^{2} q^{2} / 4\right), \quad b_{c}=1.38 \mathrm{fm},
$$

and for the valence neutrons,

$$
\rho_{v}(q)=4\left(1-b_{v}^{2} q^{2} / 6\right) \exp \left(-b_{v}^{2} q^{2} / 4\right), \quad b_{v}=1.99 \mathrm{fm}
$$

\section{IMPULSE APPROXIMATION}

The product of the target wave functions in Eq. (5) is strongly peaked about the value $P=\left|\vec{p}+\vec{p}^{\prime}\right| / 2=0$. Thus, the optical potential matrix elements will sample the $N N$ transition amplitude at and near $\vec{P}=0$. The smooth variation of the the $N N$ amplitude over the range of relevant momenta leads to the optimal factorization form of the optical potential [16]. Given the ${ }^{8} \mathrm{He}$ structure discussed above the optical potential can then be written

$$
\left\langle\vec{k}^{\prime}|U| \vec{k}\right\rangle=\frac{A-1}{A}\left[\rho_{c}(q)\left\langle\frac{1}{2}\left(\vec{k}^{\prime}+\frac{\vec{q}}{2}\right)\left|\overline{\bar{t}}_{01}(\omega)\right| \frac{1}{2}\left(\vec{k}-\frac{\vec{q}}{2}\right)\right\rangle+\rho_{v}(q)\left\langle\frac{1}{2}\left(\vec{k}^{\prime}+\frac{\vec{q}}{2}\right)\left|\bar{t}_{p n}(\omega)\right| \frac{1}{2}\left(\vec{k}-\frac{\vec{q}}{2}\right)\right\rangle\right]
$$

with $\omega=E_{0}-\hbar^{2} Q^{2} / 4 m$. Here $\overline{\bar{t}}_{01}$ is the spin and isospin average, due to the closed shell core, of the $N N$ amplitude and $\bar{t}_{p n}$ is the spin average, due to the closed valence nucleon subshell, of the $N N$ amplitude for the incident proton-struck neutron scattering. Here $\rho_{c}(q)$ and $\rho_{v}(q)$ are the densities associated with the core and valence nucleons, respectively. For a doubly closed shell nucleus then $\rho_{v}(q)=0$. In the present case $\rho_{c}(q)$ and $\rho_{v}(q)$ are given by Eqs. (11) and (12).

Following Eq. (10), the matrix elements of the optical potential are

$$
\begin{aligned}
\left\langle\vec{k}^{\prime}|U| \vec{k}\right\rangle= & \frac{A-1}{A}\left[\rho_{c}(q) \overline{\bar{t}}_{01}(\omega, \vec{q}, \vec{Q} / 2)\right. \\
& \left.+\rho_{v}(q) \bar{t}_{p n}(\omega, \vec{q}, \vec{Q} / 2)\right]
\end{aligned}
$$

or, alternatively,

$$
\begin{aligned}
\left\langle\vec{k}^{\prime}|U| \vec{k}\right\rangle= & \frac{A-1}{A}\left[\rho_{c}(q) \overline{\bar{t}}_{01}(\omega, q, Q / 2, \phi)\right. \\
& \left.+\rho_{v}(q) \bar{t}_{p n}(\omega, q, Q / 2, \phi)\right]
\end{aligned}
$$

with $\phi$ the angle between the vectors $\vec{Q}$ and $\vec{q}$. Explicitly, the central and spin-orbit components of the interaction are therefore

$$
\left\langle\overrightarrow{k^{\prime}}|U| \vec{k}\right\rangle=U_{c}\left(\overrightarrow{k^{\prime}}, \vec{k}\right)+U_{\ell s}\left(\overrightarrow{k^{\prime}}, \vec{k}\right) \vec{\sigma}_{0} \cdot \hat{n}
$$

with $\hat{n}$ the unit normal to the $N A$ scattering plane in the $N A$ c.m. frame. With the normalizations adopted in Eqs. (11) and (12) for the core and valence nucleon densities, then the central and spin-orbit optical potential form factors are

$$
\begin{aligned}
U_{c}\left(\overrightarrow{k^{\prime}}, \vec{k}\right)= & \frac{A-1}{A}\left[-\frac{\hbar^{2}}{4 \pi^{2} \mu}\right]\left[\mathcal{A}_{0}(\omega, q, Q / 2, \phi) \rho_{c}(q)\right. \\
& \left.+\mathcal{A}_{p n}(\omega, q, Q / 2, \phi) \rho_{v}(q)\right] \\
U_{\ell s}\left(\overrightarrow{k^{\prime}}, \vec{k}\right)= & \frac{A-1}{A}\left[-\frac{\hbar^{2}}{4 \pi^{2} \mu}\right]\left[\mathcal{C}_{0}(\omega, q, Q / 2, \phi) \rho_{c}(q)\right. \\
& \left.+\mathcal{C}_{p n}(\omega, q, Q / 2, \phi) \rho_{v}(q)\right]
\end{aligned}
$$

with $\mathcal{A}_{0}$ given by Eq. (10). The proton-valence nucleon amplitude $\mathcal{A}_{p n}$ is 


$$
\mathcal{A}_{p n}=\mathcal{A}_{0}-\mathcal{A}_{\tau}
$$

and similarly for $\mathcal{C}_{p n}$.

Systematic studies of the $N N$ amplitude have shown that for the central and spin-orbit terms $\mathcal{A}$ and $\mathcal{C}$, the variation of the amplitudes with the angle $\phi$ is very slow $[17,21]$. Thus, we take the on-shell value $\phi=\pi / 2$ in the evaluation of these amplitudes.

For the evaluation of the appropriate $N N$ energy parameter $\omega=E_{0}-\hbar^{2} Q^{2} / 4 m$ we make the usual assumption that the momentum of the projectile is fixed at the on-shell value $k_{0}$ and that the dominant momenta of the struck nucleon are small. These assumptions should be particularly appropriate for the longer-ranged contributions to the optical potential arising from the valence (halo) nucleons, where the nucleons interact in regions of low matter density and are of lower momenta. In this limit the $N N$ energy is assumed fixed with $\omega=E_{0} / 2$ [16], the impulse approximation. In the present assessment of the role of the spin-orbit interaction, this is the value used in the calculation of the $N N$ amplitude.

\section{DISCUSSION OF RESULTS}

We present calculations for proton- ${ }^{8} \mathrm{He}$ scattering at incident projectile energies of $72 \mathrm{MeV} /$ nucleon and 200 $\mathrm{MeV} /$ nucleon [26]. We include the proton- ${ }^{8} \mathrm{He}$ Coulomb interaction, assumed to act on the center of mass of the ${ }^{8} \mathrm{He}$, using the subtracted momentum space method discussed in Ref. [27]. The method has been shown to produce accurate calculations of scattering observables. The Coulomb interaction was calculated from the deduced ${ }^{8} \mathrm{He}$ charge density. The Coulomb effects are relatively small in the energy region of interest. The free $N N$ amplitudes were calculated exactly, on and off the energy shell, from the Paris potential $[20,22]$. Once the core and valence nucleon densities of ${ }^{8} \mathrm{He}$ are given, for example those of Eqs. (11) and (12), there are no free parameters in the calculations.

Figure 1 presents the calculated and experimental values of the differential cross section angular distribution for proton- ${ }^{8} \mathrm{He}$ scattering at a laboratory energy of 72 $\mathrm{MeV} /$ nucleon. The data were taken from Ref. [11]. The solid curve shows the results of using the full microscopic optical potential, including central and spin-orbit terms. Although our interest here is not in the detailed description of the experimental data, nevertheless, we see that the single scattering impulse approximation calculations produce a very reasonable description of the experimental points. The smallest angle scattering data are reasonably reproduced. These data points were somewhat underestimated in the Glauber model calculations of Ref. [13]. The dot-dashed curve shows the calculated cross section if we make the zero-range approximation to the $N N$ transition amplitude in the present work. The corrections due to the finite range of the $N N$ amplitude are also clearly very significant.

The dashed line in Fig. 1 also shows the full finite-range calculations but in the complete absence of the spin-orbit terms in the proton ${ }^{8} \mathrm{He}$ interaction, that is, replacing

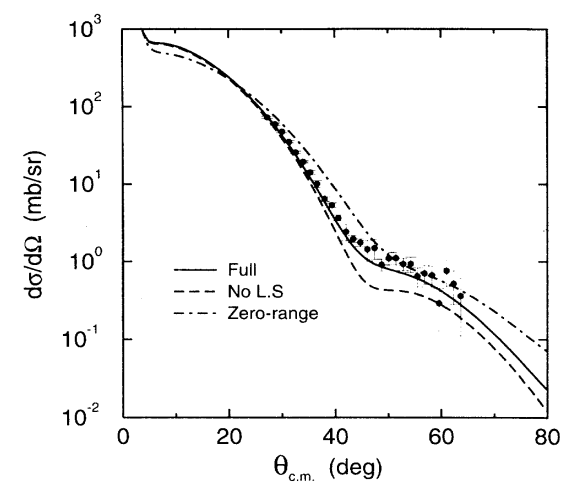

FIG. 1. Calculated and experimental [11] differential cross section angular distributions for $p-{ }^{8} \mathrm{He}$ scattering at 72 $\mathrm{MeV} /$ nucleon. The solid and dashed curves show the full microscopic optical potential calculations, including central and spin-orbit terms and in the absence of spin-orbit terms, respectively. The dot-dashed curve assumes the zero-range approximation to the $N N$ amplitude.

$\mathcal{C}_{0}=\mathcal{C}_{p n}=0$ in Eq. (18). The effects on the calculated cross section angular distribution are once again very significant, even given the accuracy of the available data. In fact the magnitude of the changes induced due to the spin dependence is of the same order as those produced when changes are made to the assumed ${ }^{8} \mathrm{He}$ density distribution [13]. Clear therefore is that analyses of the structure or extension of the valence nucleon (halo) distributions from elastic ion-nucleon data need to take account of the finite-range and spin-orbit potential effects associated with a realistic description of the $N N$ amplitude.

Figure 2 shows the corresponding differential cross section calculations at $200 \mathrm{MeV} /$ nucleon incident energy. The two curves have the same meaning as those in Fig. 1. We do not show the zero-range calculation. As might be expected, the effects of the spin-orbit interaction increase very significantly at this energy and are responsible for

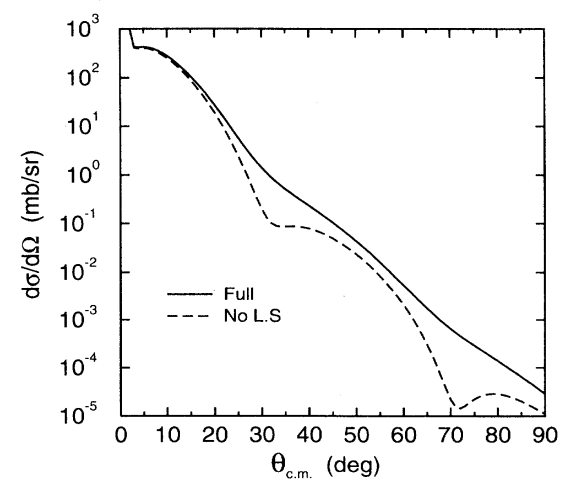

FIG. 2. Calculated differential cross section angular distributions for $p^{8} \mathrm{He}$ scattering at $200 \mathrm{MeV} /$ nucleon. The solid and dashed curves show the full microscopic optical potential calculations, including central and spin-orbit terms and in the absence of spin-orbit terms, respectively. 
the smoothing and filling in of the diffraction minima in the cross section observed in the absence of the spin-orbit term.

Having established the importance of finite-range and spin-orbit potential effects to obtaining quantitative cross section predictions, in the remainder of this section we perform additional calculations to assess the valence nucleon (halo) contributions to the central and spin-orbit component of the optical potential and scattering observables. We consider microscopic calculations where the core and valence nucleon $N N$ amplitudes, that is, the $\mathcal{C}$ and $\mathcal{A}$ in Eqs. (17) and (18), are selectively switched on and off while retaining the correct proton- ${ }^{8} \mathrm{He}$ kinematics.

In Figs. 3-6 we compare such calculations with the full physical calculations at $72 \mathrm{MeV} /$ nucleon and 200 $\mathrm{MeV} /$ nucleon. Figures 3 and 4 show the calculated differential cross section and vector analyzing power angular distributions at $72 \mathrm{MeV}$. The full calculations are shown by the solid curves. Also shown, dashed curves, are the no spin-orbit calculations for the cross section, the dashed curves of Fig. 1. The dotted and dot-dashed curves result from setting $\mathcal{A}_{p n}=\mathcal{C}_{p n}=0$ in Eqs. (17) and (18), and $\mathcal{C}_{p n}=0$ in Eq. (18), respectively. That is, the dotted curves result when the central and spin-orbit components of the optical potential arising from the interaction of the incident nucleon with the valence neutrons are removed. The dot-dashed curves result when only the spin-orbit component of the optical potential arising from the valence nucleon density is removed. The cross section displays clearly the importance of the distortion due to the valence nucleon interactions to the full calculation. We note, however, that the spin-orbit component of the optical potential arising from the valence nucleons has only a very small effect upon the cross section angular distribution. The spin dependence in the cross section thus conveys essentially no information regarding the structure of the halo nucleons and should be well determined, result-

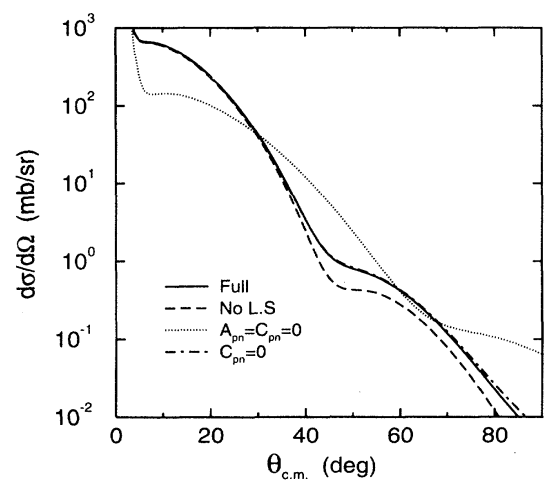

FIG. 3. Calculated differential cross section angular distributions for $p^{8} \mathrm{He}$ scattering at $72 \mathrm{MeV} /$ nucleon. The solid and dashed curves show the full microscopic calculations with and without spin-orbit terms. The dotted and dot-dashed curve result when removing the central and spin-orbit, or only the spin-orbit, valence nucleon contributions to the optical potential.

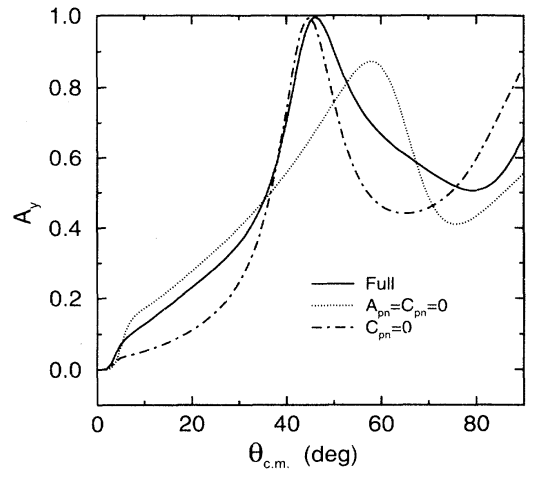

FIG. 4. Calculated vector analyzing power angular distributions for $p{ }^{8} \mathrm{He}$ scattering at $72 \mathrm{MeV} /$ nucleon. The curves are as in Fig. 3.

ing as it does, from the core of tightly bound nucleons. This is not the case for the analyzing power angular distribution where there are significant differences between the solid, dotted, and dot-dashed curves in Fig. 4 due to the valence nucleon contribution to the central and spin-dependent interactions. The conclusion is therefore, for ${ }^{8} \mathrm{He}$, while we have access only to cross section data, the required spin-dependent effects will be due almost entirely to the interaction of the target nucleon with the core. There is, however, significant sensitivity in the analyzing power angular distribution to the valence nucleon distribution, through both its central and spin-orbit potential components, and such polarization data may be important in the future in clarifying this structure.

Figures 5 and 6 show the corresponding cross section and vector analyzing power calculations at $200 \mathrm{MeV}$. The curves have the same meanings as in Figs. 3 and 4 . The conclusions regarding the importance of the core nucleon contributions to the spin-orbit interaction and the valence nucleon contributions to the central distortion upon the cross section angular distribution are confirmed at this higher energy. Again there is sensitivity in the analyzing power angular distribution to the valence nucleon distribution, through its central and spin-orbit potential

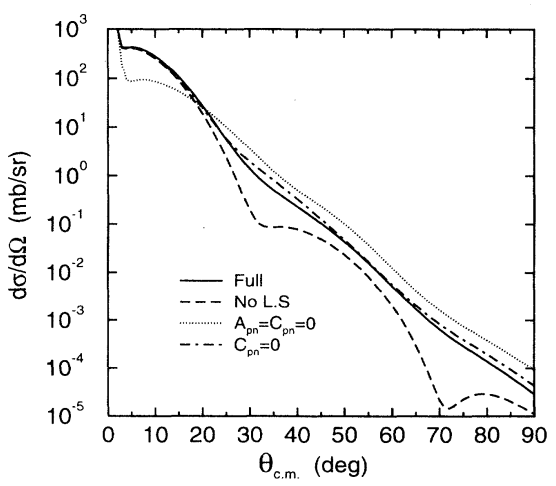

FIG. 5. Calculated differential cross section angular distributions for $p-{ }^{8} \mathrm{He}$ scattering at $200 \mathrm{MeV} /$ nucleon. The curves are as in Fig. 3. 


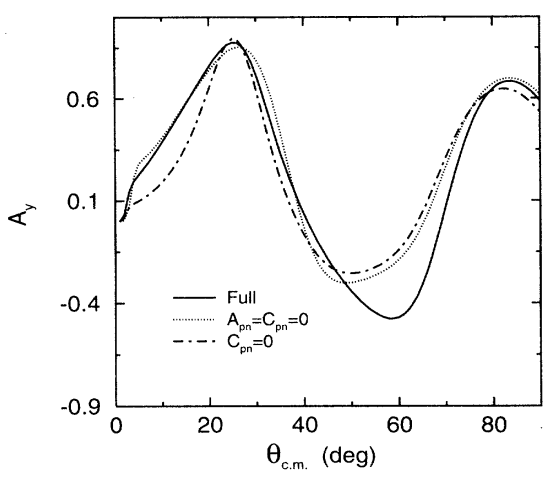

FIG. 6. Calculated vector analyzing power angular distributions for $p^{8}{ }^{8} \mathrm{He}$ scattering at $200 \mathrm{MeV} /$ nucleon. The curves are as in Fig. 4.

contributions.

Even at $72 \mathrm{MeV}$, it is evident that the spin-dependent effects on the cross section are significant. The calculations reveal, however, that the valence nucleon contribution to the spin-orbit interaction has very little effect upon this observable. However, the valence nucleon contribution to the spin-orbit interaction is manifest in the calculated analyzing powers. It would thus appear that polarization data, such as would be acquired from ${ }^{8} \mathrm{He}$ scattering from a polarized proton target, could provide additional information on the valence neutron distribution. These results, regarding the importance of the spin dependence to the calculated cross sections, become more significant with increasing nucleon energy.

\section{CONCLUSIONS}

The microscopic optical potential for proton elastic scattering from ${ }^{8} \mathrm{He}$ is evaluated using the single scatter- ing approximation to the KMT multiple scattering formalism. This includes automatically the finite range of the $N N$ amplitude and the central and spin-orbit components of the optical potential arising from the spin dependence of the free $N N$ amplitude. Calculations are presented for 72 and $200 \mathrm{MeV} /$ nucleon incident energies. The magnitudes of the effects, on the calculated differential cross sections and analyzing powers, of the halo structure in ${ }^{8} \mathrm{He}$, and of the spin dependence of the optical potential, are investigated. The elastic scattering observables are found to be sensitive to both the inclusion of the finite range and the spin dependence of the elementary $N N$ amplitude. It follows that analyses which attempt to determine information on the structure or radial extent of the valence nucleon (halo) distributions from such data must take into account these effects if quantitative conclusions are to be made. While the calculated cross section angular distributions are sensitive to the spin dependence due to the core nucleons, and to the contribution of the valence nucleons to the central distortion, they are insensitive to the diffuse spin-orbit term generated by the valence particles. This is not true of the calculated analyzing powers in which the spin dependence due to the valence nucleons is manifest. The available cross section data at $72 \mathrm{MeV}$ [11] appear consistent, within the present calculations, with the COSMA ${ }^{8} \mathrm{He}$ density distribution of Zhukov et al. [25].

\section{ACKNOWLEDGMENTS}

Financial support of the United Kingdom Engineering and Physical Sciences Research Council (EPSRC) in the form of Grants Nos. GR/J95867, GR/K33026, and GR/H89678 (for R.C.) is gratefully acknowledged.
[1] Proceedings of the First International Conference on Radioactive Nuclear Beams, Berkeley, CA, edited by W.D. Meyers, J.M. Nitschke, and E.B. Noeman (World Scientific, Singapore, 1990).

[2] I. Tanihata et al., Phys. Lett. 160B, 380 (1985); I. Tanihata, H. Hamaguchi, O. Hashimoto, Y. Shida, N. Yoshikava, K. Sugimoto, O. Yamakawa, T. Kobayashi, and N. Takahashi, Phys. Rev. Lett. 55, 2676 (1985).

[3] I. Tanihata, T. Kobayashi, O. Yamakawa, S. Shimoura, K. Ekuni, K. Sugimoto, N. Takahashi, T. Shimoda, and H. Sato, Phys. Lett. B 206, 592 (1988).

[4] M. Fukuda et al., Phys. Lett. B 268, 339 (1991); A.C. Villari et al., ibid. 268, 345 (1991).

[5] I. Tanihata, Nucl. Phys. A522, 275c (1990).

[6] I. Tanihata, D. Hirata, T. Kobayashi, K. Sugimoto, and H. Toki, Phys. Lett. B 289, 261 (1992).

[7] G.R. Satchler, K.W. McVoy, and M.S. Hussein, Nucl. Phys. A522, 621 (1991).
[8] M.C. Mermaz, Phys. Rev. C 47, 2214 (1993).

[9] I.J. Thompson, J.S. Al-Khalili, J.A. Tostevin, and J.M. Bang, Phys. Rev. C 47, R1364 (1993); J.S. Al-Khalili and J.A. Tostevin, ibid. 49, 386 (1994); J.S. Al-Khalili, I.J. Thompson, and J.A. Tostevin, Nucl. Phys. A581, 331 (1995).

[10] C.B. Moon, M. Fujimaki, S. Hirenzaki, N. Inabe, K. Katori, J.C. Kim, T. Kobayashi, T. Kubo, H. Kumagai, S. Shimoura, T. Suzuki, and I. Tanihata, Phys. Lett. B 287, 39 (1992).

[11] A.A. Korsheninnikov et al., Phys. Lett. B 316, 38 (1993).

[12] A.N.F. Aleixo, C.A. Bertulani, and M.S. Hussein, Phys. Rev. C 43, 2722 (1991).

[13] L.V. Chulkov, C.A. Bertulani, and A.A. Korsheninnikov, Nucl. Phys. A (to be published).

[14] A.K. Kerman, H. McManus, and R.M. Thaler, Ann. Phys. (N.Y.) 8, 551 (1959).

[15] H.F. Arellano, F.A. Brieva, and W.G. Love, Phys. Rev. 
C 41, 2188 (1990); H.F. Arellano, W.G. Love, and F.A. Brieva, ibid. 43, 2734 (1991).

[16] R. Crespo, R.C. Johnson, and J.A. Tostevin, Phys. Rev. C 41, 2257 (1990).

[17] R. Crespo, R.C. Johnson, and J.A. Tostevin, Phys. Rev. C 44, R1735 (1991), 46, 279 (1992).

[18] Ch. Elster, T. Cheon, E.F. Redish, and P.C. Tandy, Phys. Rev. C 41, 814 (1990); C.R. Chinn, Ch. Elster, and R.M. Thaler, ibid. 44, 1569 (1991).

[19] C.R. Chinn, Ch. Elster, R.M. Thaler, and S.P. Weppner, Vanderbilt University Report, 1994.

[20] M. Lacombe, B. Loiseau, J.M. Richard, R. Vinh Mau, J. Cõté, P. Pires, and R. de Tourreil, Phys. Rev. C 21, 861 (1980).
[21] E.F. Redish and K. Stricker-Bauer, Phys. Rev. C 36, 513 (1987).

[22] An extension of the computer programs of E.F. Redish (unpublished).

[23] R. Crespo, R.C. Johnson, J.A. Tostevin, R.S. Mackintosh, and S.G. Cooper, Phys. Rev. C 49, 1091 (1994).

[24] L. Wolfenstein and J. Ashkin, Phys. Rev. 85, 947 (1952).

[25] M.V. Zhukov, A.A. Korsheninnikov, and M.H. Smedberg, Phys. Rev. C 50, R1 (1994).

[26] An extension of the program LPOTP, M.J. Paez, M.E. Sagen, and R.H. Landau, Comput. Phys. Commun. 52, 141 (1988).

[27] R. Crespo and J.A. Tostevin, Phys. Rev. C 41, 2615, (1990). 